\title{
REIKI und chronische Schmerzen
}

Durch Harmonisierung der Körperenergien kann eine Reduktion der Beschwerden erreicht werden. Von Dr. Leo Spindelberger

Der Begriff REIKI wurde Anfang des vorigen Jahrhunderts vom Jadeutet einerseits bensenergie" und ist andererseits die Bezeichnung für eine heute weltweit bekannte energetische Methode. Sie baut auf den Erfahrungen und Grundlagen der Traditionellen Chinesischen Medizin (TCM) auf und hat die Harmonisierung der Körperenergien und den freien Fluss der Lebensenergie durch den Organismus zum Ziel.

\section{Das Konzept der Lebensenergie}

Die Anerkennung einer alles durchdringenden Lebensenergie ist keine Glaubensfrage, sondern erfahrene Realität in praktisch allen großen Kulturen dieser Welt. Diese Energie wurde und wird als lebensnotwendig betrachtet, und ohne sie könnte weder der Körper, noch der Verstand existieren. Da nach dieser Auffassung auch Krankheiten wenigstens zum Teil aus dem energetischen Bereich entspringen, können die Ursachen nicht ausschließlich im Materiellen gefunden werden. Alle Heiltraditionen diese Kulturen beruhen auf der Erfahrung, dass für das Gesundsein des Menschen der freie Fluss der Lebensenergie von entscheidender Bedeutung ist Daher kennen sie alle auch Methoden, die Lebensenergie aufzubauen, sie zu harmonisieren und zum Fließen zu bringen. In den ursprünglichen Kulturen und Traditionen wurde kein Unterschied zwischen Körper, Seele und Geist gemacht. Alles war Ausdruck einer einzigen lebendigen Kraft, die verschiedenen Namen hatte:

- bei den Griechen hieß sie

PNEUMA

bei den Hindus PRANA

bei den Tibeter LUNG

- bei den Chinesen QI

- bei den Japanern KI

in der Bibel ODEM GOTTES

bei den Sufis BARAKA

in der Alchemie Europas VIS

VITALIS

- in Afrika MINGO

- in Polynesien MANA

bei den Mayas LIL

Wilhelm Reich nannte sie ORGON

moderne Physiker nennen sie NULL-PUNKT-ENERGIE

Bei REIKI werden der Energieausgleich und die Auflösung von Blockaden durch das sanfte Berühren mi den Händen bewirkt (siehe Abb.), und physiologisch lassen sich folgende Wirkweisen feststellen:

\section{1. lokale Wirkung}

Durch die sanfte Berührung mit den Händen kommt es an den entsprechenden Positionen zu eine Erwärmung mit einer begleitenden Durchblutungssteigerung, die fühl- und messbar ist.
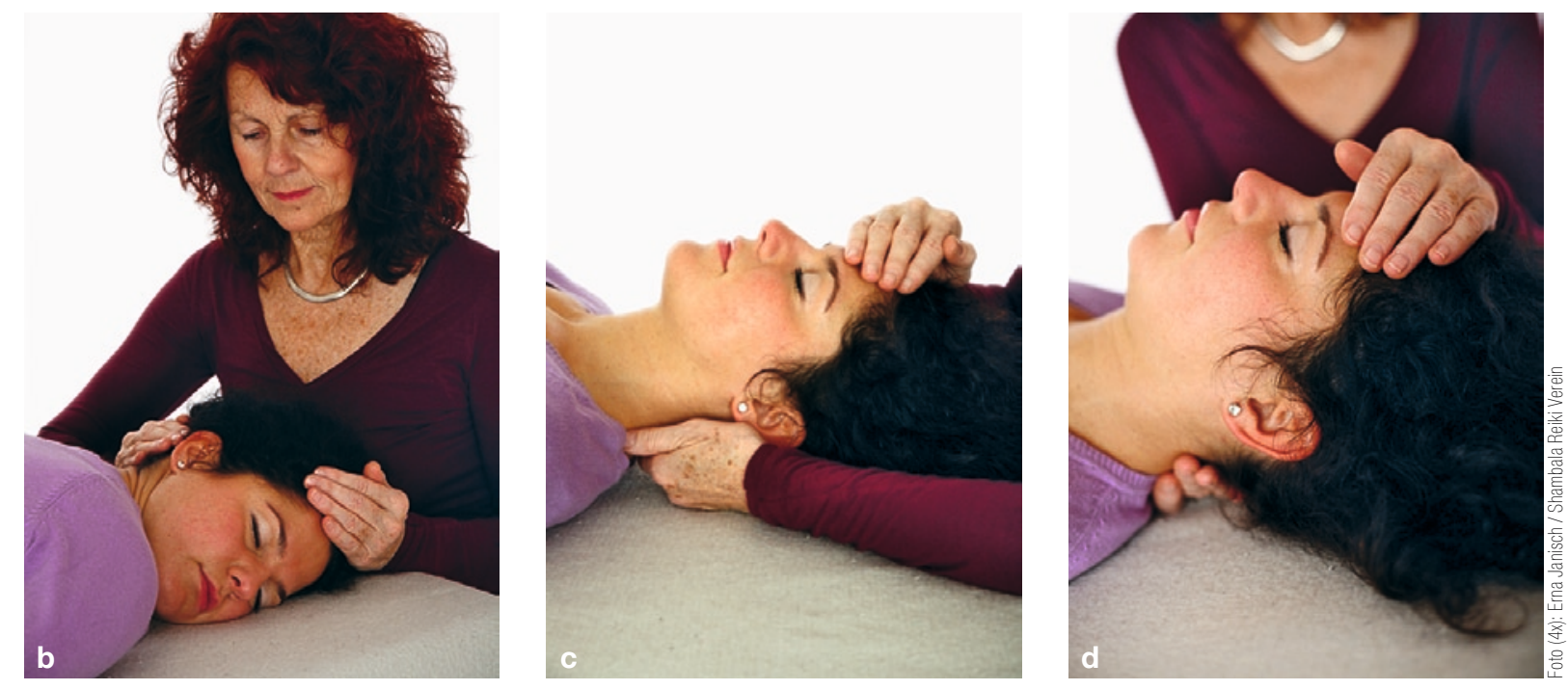

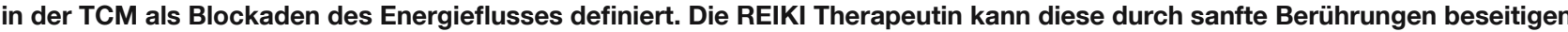
a. seitliche Kopfposition b. Stirn Nacken Position in Bauchlage c. Stirn Nacken Position in Rückenlage d. Stirn Nacken Position seitlich

Änderung des Spannungszustandes der unter den Händen liegenden Muskulatur $=$ Entspannung verspannter Muskulatu

Energieausgleich, d.h. Energieanstauungen werden abgeleitet, Energiemangel wird ausgeglichen (entspricht der Wirkung von Akupunktur, Akupressur bzw. von Shiatsu) Vermittlung von Geborgenheit durch die direkten Berührung un den sanften und einfüh Körperkontakt

Aktivierung des Parasympathikus und der Regeneration

\section{2. segmentale Wirkung}

Über Reflexbögen kommt es in den zu dem berührten Hautsegment gehörigen Erfolgsorganen zu einer Durchblutungssteigerung und dadurch

zu einer Anregung der Aktivität (= Stärkung der Funktion)

$\mathrm{zu}$ einer Beschleunigung der Regeneration

Regulation des Muskeltonus in den zum Segment gehörigen Muskeln

3. generelle Wirkungen über das Nervensystem

tiefe körperliche und emotionelle = psychische) Entspannung

Lösung von Energieblockaden und Förderung des Energieflusse Anregung der Selbstheilungskräfte und des Regenerationsvermögens

Verbesserung des Schlafes (sowohl bei Einschlaf- als auch Durchschlafstörungen)

Verbesserung der Leistungsfähigkeit und Reduktion von Müdigkeit und Kälteempfindlichkeit

Erhöhung der Stresstoleranz und Widerstandskraft gegen diverse Belastungen

Schmerzen werden in der TCM im mer als Blockaden des Energieflusse definiert. So war es nahe liegend, eine Untersuchung mit REIKI bei chronischen Schmerzzuständen durchzuführen. Durch die positiven Erfahrungen mit REIKI bei gestressten Menschen war beruflicher Stress ein wesentliche Voraussetzung für die Teilnahme an der Untersuchung. Da psychische Belastungen, Zeitdruck und Stress sehr häufig zu Verspannungen führen und diese wiederum den Energiefluss blockieren, gibt es einen engen Zusammenhang zwischen der Arbeitssituation, Stres und chronischen Schmerzzustönden. undch Chronische Beschwerden am Bewe-
gungsapparat sind gleichzeitig die häufigsten Ursachen für Krankenstände in der heutigen Arbeitswelt und arbeitsmedizinische Studien zeigen, dass es sich immer mehr um psychische Belastungen handelt, unter denen arbeitende Menschen zunehmend leiden.

Hier eine kurze Zusammenfassung der 2. REIKI Studie, die im letzten Jahr von Mitgliedern des SHAM BHALA REIKI ${ }^{\circledR}$ Vereins unter ärztlher Kontrolle durchgeführt wurde:

\section{Gegenstand der Studie}

Chronische Schmerzen im Bereich des Bewegungsapparates, mit ausgelöst durch beruflichen Stress

durchschnittliche Dauer de Schmerzen ca. zehn Jahre (1-30 Jahre)

häufigste Krankenstandsursachen im Berufsleben

\section{Ziel und Design der Studie}

Hypothese der Studie: Reiki wirkt verbessernd bei chronischen Schmerzen am Bewegungsapparat, mit ausgelöst durch Stress am Arbeitsplatz.

Art der Studie: Erhebung, Evaluierung und Dokumentation chronischer Schmerzen mittels spezi-

\section{Fazit für die Praxis}

Zusammenfassend kann man sagen, dass REIKI bei chronischen Schmerzzuständen, die im Zusammenhang mit Stress stehen, eine ähnlich gute Wirkung zeigt wie Akupunktur. Dabei ist interessant, dass viele Probanden als positiven Nebeneffekt angegeben haben, dass sie sich grundsätzlich besser entspannen können und auch viel besser schlafen. Diese Wirkungen lassen sich auf eine Aktivierung des parasympathischen Nervensystems zurückführen, was wiederum die generelle Regeneration fördert, sodass alltägliche Stresssituationen auch wieder besser gemeistert werden können. Um diese Effekte allerdings erzielen zu können, braucht es einen größeren Zeitaufwand, der im normalen medizinischen und ärztlichen Alltag kaum aufzubringen ist. Die Zusammedizinischen und ärztlichen Alltag kaum aufzubringen ist. Die ZusamÖBRT - Österreichische Berufsverband der diplomierten Reiki Therapeuten zertifiziert hat, ist eine gute Ergänzung und Möglichkeit bei den vorliegenden und in diesem Artikel beschriebenen Beschwerdebildern.
- 5 x HWS + LWS

- $6 x$ Schulter

\section{Therapeuten}

Die Probanden wurden von 20 (16 Frauen + 4 Männer) diplomierten REIKI-Therapeuten desShambhala REIKI ${ }^{\oplus}$ Vereins betreut.

Es kamen ein bis drei Personen auf einen Therapeuten

9. Abnahme der Schmerzintensität in absoluten Zahlen

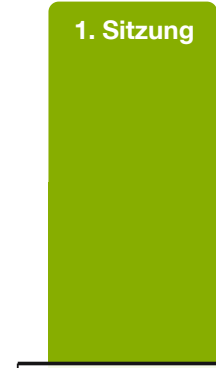

125,6

\section{Zusammenfassung}

Hypothese der Studie: Reiki wirk verbessernd bei chronischen Schmerzen am Bewegungsapparat, mit ausgelöst durch Stress am Arbeitsplatz

Ergebnis der Studie: Abnahme der Schmerzintensität um durchschnittlich 65 Prozent innerhalb von zwei Monaten

Es ist geplant, nach einem Jahr eine Nachuntersuchung durchzuführen, um auch über die Nachhaltigkeit und Langzeitwirkung Aussagen machen zu können. Derzeit beginnt gerade eine weitere Untersuchung zum Thema Schlafstörungen, da sich gezeigt hat, dass viele Teilnehmer an der Schmerzstudie sozusagen als positive "Nebenwirkung“ festgestellt haben, dass sie wieder besser schlafen können. Die Ergebnisse dieser Untersuchung werden im Sommer 2010 präsentiert werden (siehe auch: www.shambhala-reiki.at)

\section{Korresponden}

Dr. med. Leopold Sp
Habsburgergasse 10 